(KO) mice were bred to generate homozygous KOs and mouse articular cartilage was isolated to culture chondrocytes.

Results: miR-34a expression was significantly elevated in the plasma as well as cartilage and synovium of TKR patients (KL IV) compared to healthy controls and early OA patients (KL-I), respectively. Similarly, miR-34a was significantly overexpressed in mouse knee joints (cartilage and synovium) at 10 weeks post OA surgery compared to sham. To identify the biological effects of miR-34a on chondrocyte and synovial fibroblast (SF), functional studies were conducted invitro. Chondrocytes treated with miR-34a mimic had a significant reduction of SIRT1 (a direct target of miR-34a), anabolic (type II collagen and aggrecan) and autophagy markers, as well as, elevated catabolic markers (MMP13), suggesting that miR-34a contributes to cartilage degeneration. Chondrocytes treated with mir-34a inhibitor reversed these destructive effects. SFs treated with miR-34a mimic expressed elevated inflammatory (TNF- $\alpha$, IL-6), fibrotic (TGF- $\beta$, Type 1 Collagen), and autophagy markers, suggesting that miR-34a is involved in mediating synovial inflammation and fibrosis. SFs treated with miR-34a inhibitor reversed these effects.

In vivo, intra-articular injection of miR-34a mimic induced cartilage damage, loss of proteoglycan content, and elevated cell death markers (PARP p85 and Caspase 3 ) in the articular cartilage. To confirm the destructive effects of miR-34a in the articular cartilage, we used miR-34a KO mice. MiR-34a KO mice further confirmed that genetic ablation of miR-34a resulted in marked elevation in the expression of anabolic markers (type II collagen and aggrecan) and decreased expression of catabolic ADAMTS-5 in the articular cartilage.

To test the therapeutic potential of blocking miR-34a, we intra-articularly injected mir-34a inhibitor in mice subjected to OA surgery. Results showed marked reduction in the severity of cartilage degeneration in mice treated with miR-34a inhibitor. Conclusion: This study, for the first time, demonstrates miR-34a as a crucial mediator involved in OA pathogenesis and as a potential therapeutic target for limiting cartilage destruction during $\mathrm{OA}$.

Disclosure of Interests: None declared

DOI: 10.1136/annrheumdis-2019-eular.2677

\section{OP0019-HPR A SYSTEMATIC REVIEW AND META-ANALYSIS ASSESSING GASTROINTESTINAL, LIVER, RENAL AND CARDIOVASCULAR ADVERSE EVENTS OF PARACETAMOL}

Jaspreet Kaur, Burak Kundaki, Georgina Nakafero, Abhishek Abhishek, Michael Doherty, Weiya Zhang. University of Nottingham, Academic Rheumatology, Nottingham, United Kingdom

Background: Despite its modest efficacy, guidelines consistently recommend paracetamol as a first-line analgesic for osteoarthritis (OA) based on its perceived safety. However, there is growing controversy, highlighted in the National Institute for Health and Care Excellence OA (NICE) 2014 guidance on OA, that paraceta$\mathrm{mol}$ is not as safe as previously thought, especially at the highest therapeutic dose of $4 \mathrm{gm} /$ day.

Objectives: To investigate the association between paracetamol and gastrointestinal, liver, renal and cardiovascular adverse events both in randomised controlled trials (RCTs) and observational studies.

Methods: We systematically searched MEDLINE, EMBASE, PUBMED, AHMED, CINAHL, Web of Science, and Google Scholar for published literature in any language to the end of November 2018 for (1) RCTs of paracetamol in symptomatic $\mathrm{OA}$, and (2) observational studies irrespective of any underlying condition, to determine the risk of gastrointestinal, liver, renal and cardiovascular adverse events. We included studies assessing oral paracetamol in people aged $\geq 18$ years and reporting on clinically relevant adverse effects. Risk ratio (RR) and $95 \%$ confidence interval $(\mathrm{Cl})$ were estimated for RCT and cohort studies, whereas odds ratio $(\mathrm{OR})$ and $95 \% \mathrm{Cl}$ were used for case-control studies. Results were pooled as appropriate using random effects model. The risk of bias was assessed using modified Cochrane tool for RCTS and Newcastle Ottawa scale for observational studies.

Results: We reviewed titles and abstracts of 3,622 records in the systematic search (1,997 RCTs and 1,635 observational studies). After examining full papers 23 RCTs (7,863 participants), 15 cohort studies (2,262,517 participants) and 34 case-control studies $(441,638$ participants) met inclusion criteria.

Compared to placebo, paracetamol was associated with increased incidence of treatment-related adverse events (RR $1.35,95 \% \mathrm{Cl} 1.04$ to 1.75 ), especially diarrhoea (RR 2.14, 95\% Cl 1.35 to 3.37) and abnormal liver function (RR 3.99, 95\% $\mathrm{Cl} 2.05$ to 7.77). The pooled OR from twelve age and gender matched case-control studies (7894 participants) was 1.36 (95\% Cl 1.13 to 1.64$)$ for upper gastrointestinal bleeding. In addition, a dose response relationship was observed in a cohort study $(382,404$ participants) for this outcome. The RR was 1.11 (95\% Cl 1.04 to 1.21 ) with low dose paracetamol (measured as medication possession rate) and $1.49(95 \% \mathrm{Cl} 1.29$ to 1.71$)$ with high dose paracetamol. Paracetamol was not associated with cardiovascular events in two RCTs (775 participants)
(RR 1.27, 95\% Cl 0.06 to 27.77 ) and three case-control studies (42,180 participants) (OR $0.97,95 \% \mathrm{Cl} 0.77$ to 1.21$)$, but in three cohort studies $(208,926$ participants) (RR $1.35,95 \% \mathrm{Cl} 1.14$ to 1.59). There were insufficient data in RCTs and case-control studies for renal adverse events. However, three cohort studies (7,360 participants) demonstrated a dose response relationship between parace tamol and renal function impairment (defined as $\geq 30$ percentage decrease in estimated glomerular filtration rate). The data of these three cohort studies could no be pooled due to different doses of paracetamol. One cohort study (1697 participants) reported $\mathrm{RR}$ of $1.40(95 \% \mathrm{Cl} 0.79$ to 2.48$)$ for renal impairment for paracetamol $100-499 \mathrm{~g}$, and 2.19 (95\% Cl 1.4 to 3.43 ) for paracetamol $>3000 \mathrm{~g}$.

Conclusion: Paracetamol is associated with diarrhoea and abnormal liver function in short-term trial data, and with upper Gl bleeding and renal impairment in long-term observational data. Methodological limitations of observational data such as channelling bias, may confound the results. Large-scale well-designed prospective studies are needed to ascertain the long-term safety of paracetamol. Disclosure of Interests: Jaspreet Kaur: None declared, Burak Kundaki: None declared, Georgina Nakafero: None declared, Abhishek Abhishek Grant/research support from: AstraZeneca and Oxfordlmmunotech, Grant/research support from: AstraZeneca and Oxford Immunotech, Speakers bureau: Menarini pharmaceuticals, Speakers bureau: Menarini pharmaceuticals, Michael Doherty Grant research support from: AstraZeneca funded the Nottingham Sons of Gout study Consultant for: Advisory Boards on Grunenthal and Mallinckrodt, Weiya Zhang Consultant for: Grunenthal for advice on gout management, Speakers bureau: Bioiberica as an invited speaker for EULAR 2016 satellite symposium DOI: 10.1136/annrheumdis-2019-eular.2802

\section{OP0020 LESS IS MORE: ANA-LYSING THE IMPACT OF REPEATED ANTINUCLEAR ANTIBODY TESTING}

$\underline{A i ~ L i ~ Y e o ~}^{1,2,3}$, Jason Ong ${ }^{3}$, Kathryn Connelly ${ }^{1}$, Suong Le ${ }^{2}$, Ronnie Ptasznik ${ }^{2}$, Jane Ross ${ }^{2}$, Eric F. Morand ${ }^{1,3}$, Michelle Leech ${ }^{1,3}$. ${ }^{1}$ Monash Health, Department of Rheumatology, Melbourne, Australia; ${ }^{2}$ Monash Health, Department of Informatics, Melbourne, Australia; ${ }^{3}$ Monash University, Melbourne, Australia

Background: Minimising unnecessary tests is a global health economic priority with multiple initiatives in place to avoid inappropriate healthcare utilisation(1) and harm. Anti-nuclear antibody (ANA) testing is frequently performed as a diagnostic test for autoimmune conditions, such as systemic lupus erythematosus (SLE) or as a screening test in patients with inflammatory or musculoskeletal symptoms. The value of serial testing in the monitoring of such conditions is unclear and false positive tests can lead to unnecessary further investigation and increased patient anxiety (2)

Objectives: To evaluate the frequency of repeated ANA testing as a prelude to Electronic Medical Record (EMR) test alert design in an Australian healthcare network. The primary endpoint was calculation of the total cost associated with repeated testing and whether a longitudinal change in ANA resulted in any new ANA associated rheumatological diagnoses. Our secondary endpoint was the examination of baseline ANA testing behaviours.

Methods: We retrospectively analysed data from a multi-centre tertiary health network in Melbourne, Australia across a 7-year period (19 March 2011 to 23 July 2018). ANA and other autoimmune test results were obtained from the hospital pathology system with a positive ANA cut off set at 1:160. Clinical information was sourced from clinical information systems on patients who had a change in ANA result from negative to positive on repeat testing. The associated cost of repeated ANA testing was calculated based on the baseline cost to the public system.

Results: A total of 36,715 ANA tests (excluding 980 cancelled same-day requests) were performed in 28,840 patients. Of these, $14,058(38.3 \%)$ were positive with females accounting for $9,265(65.9 \%, p<0.001)$. The most frequent ANA patterns were homogenous (47.4\%) and nucleolar (23.3\%). ANA titres were as follows; $1: 160$ (41.4\%), 1:320 (15.3\%), 1:640 (13.1\%) and 1:1280 (29.2\%). 7,875 $(21.4 \%)$ of tests were repeat tests. Of these $511(6.5 \%)$ results changed from neg ative to positive. The median time between a negative ANA result to the first positive result was 1.71 years (IQR 0.50-3.55). Clinical information was captured for a median duration of 1.24 years (IQR $0.50-2.07$ ) following a positive ANA result. A change to positive ANA was associated with a new ANA-associated rheumatolog ical diagnosis in only 5 cases ( 2 SLE, 1 scleroderma and 2 undifferentiated connective tissue disease) with a positive predictive value calculated at 0.01 . When comparing patients who with a new diagnosis to those with no new diagnosis, there was no difference between ANA titre, pattern, duration to first positive ANA ordering location or clinician, or age of first positive ANA test. The direct total cost for the government of all ANA testing was AUD $\$ 903,189$, of which repeat testing contributed AUD\$193,725.

Conclusion: Repeat ANA testing after a negative result had limited utility in the diagnosis of ANA associated rheumatological conditions with a positive predictive value of only 0.01 , and resulted in high cost. New technology and clinical alert systems may help reduce unnecessary testing with potential significant direct cost savings when extrapolated across the Australian healthcare system. 
REFERENCES:

[1] Levinson W, Kallewaard M, Bhatia RS, Wolfson D, Shortt S, Kerr EA. 'Choosing Wisely': a growing international campaign. BMJ Qual Saf. 2015;24(2):167-74.

[2] Abeles AM, Abeles M. The clinical utility of a positive antinuclear antibody test result. The American journal of medicine. 2013;126(4):342-8.

Disclosure of Interests: Ai Li Yeo: None declared, Jason Ong: None declared, Kathryn Connelly: None declared, Suong Le: None declared, Ronnie Ptasznik: None declared, Jane Ross: None declared, Eric F. Morand Grant/research support from: AstraZeneca, Bristol Myers Squibb, Janssen, Merck Serono, and UCB, Consultant for: AstraZeneca, Eli Lilly, Janssen, and Merck Serono, Speakers bureau: AstraZeneca, Michelle Leech: None declared DOI: 10.1136/annrheumdis-2019-eular.4517

\begin{tabular}{|l|l}
\hline OP0021 & PREDICTING SEVERE INFECTION IN REPEAT CYCLES \\
OF RITUXIMAB AND EFFECTS OF \\
HYPOGAMMAGLOBULINAEMIA FOR THE TREATMENT \\
OF RHEUMATIC AND MUSCULOSKELETAL DISEASES
\end{tabular}

Md Yuzaiful Md Yusof ${ }^{1,2}$, Edward Vital ${ }^{1,2}$, Damien M. Mcelvenny ${ }^{3}$,

Elizabeth Hensor ${ }^{1,2}$, Sudipto Das ${ }^{1,2}$, Shouvik Dass ${ }^{1}$, Andy C. Rawstron ${ }^{4}$, Maya Buch ${ }^{1,2}$, Paul Emery ${ }^{1,2}$, Sinisa Savic ${ }^{1,2}$. ${ }^{1}$ University of Leeds, Leeds Institute of Rheumatic and Musculoskeletal Medicine, Leeds, United Kingdom; ${ }^{2}$ Leeds Teaching Hospitals NHS Trust, NIHR Leeds Biomedical Research Centre, Leeds, United Kingdom; ${ }^{3}$ University of Manchester, Institute of Population Health, Manchester, United Kingdom; ${ }^{4}$ Leeds Teaching Hospitals NHS Trust, Haematological Malignancy Diagnostic Service, Leeds, United Kingdom

Background: Rituximab (RTX) is effective in treating various rheumatic and musculoskeletal diseases (RMDs). Repeat cycles are often required for disease control but may lead to hypogammaglobulinaemia. Low IgG at baseline has been associated with increased risk of severe infection event (SIE) post-RTX. However, there are limited data on predictors of SIEs in repeat cycles including immunoglobulin levels and B-cell numbers as well as outcomes of hypogammaglobulinaemia.

Objectives: To assess predictors of SIEs in repeat RTX cycles and effects of hypogammaglobulinaemia in terms of SIEs rates, humoral response and its persistence post-cessation of RTX.

Methods: A retrospective study was conducted in the first 700 consecutive ARD patients treated with at least a cycle of RTX in Leeds. IgM, IgA and IgG levels were measured at baseline and 4-6 months after each cycle. For cycles 2-4 (C24 ), predictors for SIEs were analysed using mixed-effects logistic regression analysis.

Results: 550 patients were female, mean(SD) age 56(16) years and median (IQR) disease duration 7.9(3.4-15.0) years. 507(72\%) had RA, 94(13\%) SLE, 49 (7\%) AAV, 14(2\%) inflammatory myopathies, 9(1\%) pSS, 5(1\%) APS, 6(1\%) SSc and $16(3 \%)$ other CTDs. $364(52 \%)$ were biologic-naïve and $514(73 \%)$ were on concomitant DMARDs. Total follow-up: 2880 patient-years (PY). 281 SIEs were recorded in 176 patients (9.8/100 PY). In C1, we had validated that low IgG was predictive of SIE within 12 months of $\mathrm{C} 1$. For cycles 2-4, in multivariable analysis, non-RTX-specific comorbidities [chronic lung OR $(95 \% \mathrm{Cl}) 2.4$ (1.3-4.4), diabetes 2.9 (1.2-6.9), heart failure $6.3(1.4-28.1)$, previous cancer 3.0 (1.3-6.7) and severe infection 6.3 (3.0-13.4)] and RTX-specific variables [higher corticosteroid dose 1.08 (1.02-1.14), higher IgM 1.3 (1-1.7) and longer retreatment time 1.01 (1-1.02)] were associated with increased odds of SIEs, but not B-cell numbers or depletion status. Higher IgG reduced the risk 0.88 (0.8-0.96). Of 103 patients with low IgG for at least 4 months duration, SIEs rates were higher in those with low baseline $\operatorname{lgG}(16.4 \mathrm{PY})$ or acquired it during/post-RTX (21.3 PY) versus those with normal IgG (9.7 PY), 5/8(64\%) had impaired humoral response to pneumococcal and haemophilus following vaccination challenge and only 4/11(36\%) had IgG normalised after switching therapies. Overall, $7(1 \%)$ of the patients required Ig replacement based on recurrent sino-pulmonary SIEs and/or low lgG.

Conclusion: Immunoglobulin should be monitored at baseline and before each RTX cycle to identify patients at risk of SIEs. Vigilance is needed for those with lower $\lg$ as this is a consistent predictor of SIE and may affect infection outcomes when patients are switched to a different bDMARD. For those at risk of SIEs, reduction of corticosteroid dose could reduce risk. Low B-cell numbers were not predictive of SIEs.

Acknowledgement: This research was supported by Octapharma and NIHR (DRF-2014-07-155). The views expressed are those of the author(s) \& not necessarily the NHS, NIHR or DOH.

Disclosure of Interests: Md Yuzaiful Md Yusof: None declared, Edward Vital Grant/research support from: He has received honoraria and research grant support from Roche, GSK and AstraZeneca., Damien M McElvenny: None declared, Elizabeth Hensor: None declared, Sudipto Das: None declared, Shouvik Dass Grant/research support from: Roche and GSK,
Andy C Rawstron: None declared, Maya Buch Grant/research support from: Pfizer LTD, UCB, Consultant for: AbbVie, Eli Lilly, EMD Serono, Pfizer Ltd., Sanofi, Paul Emery Grant/research support from: Pfizer, MSD, AbbVie Bristol-Myers Squibb, Roche, Consultant for: Pfizer, MSD, AbbVie, BristolMyers Squibb, UCB, Roche, Novartis, Gilead,Samsung, Sandoz and Lilly, Sinisa Savic Grant/research support from: Novartis and Sobi DOI: 10.1136/annrheumdis-2019-eular.7573

\section{OP0022 \\ DO MRI-DETECTED EROSIONS IN PATIENTS WITH CLINICALLY SUSPECT ARTHRALGIA PREDICT PROGRESSION TO RHEUMATOID ARTHRITIS? A LONGITUDINAL STUDY}

Fenne Wouters $^{1}$, Xanthe Matthijssen ${ }^{1}$, Debbie Boeters ${ }^{1}$, Robin Ten Brinck ${ }^{1}$, Annette van der Helm - van Mil ${ }^{1,2}$, Ellis Niemantsverdriet ${ }^{1} .{ }^{1}$ Leiden University Medical Centre, Rheumatology, Leiden, Netherlands; ${ }^{2}$ Erasmus University Medical Centre, Rheumatology, Rotterdam, Netherlands

Background: Radiographic joint erosions are a hallmark of Rheumatoid Arthritis (RA). MRI is more sensitive than radiographs in detecting erosions. It is unknown if MRI-detected erosions are predictive for RA-development in patients with Clinically Suspect Arthralgia (CSA).

Objectives: We investigated the prognostic value of MRI-detected erosions (any $\mathrm{MRI}$-erosion, or MRI-erosion characteristics that were recently identified as specific for RA) in CSA.

Methods: Patients presenting with CSA $(n=491)$ underwent contrast-enhanced 1.5T MRI of the wrist, metacarpophalangeal (MCP) and metatarsophalangeal (MTP) joints at baseline. MRIs were scored according to RAMRIS. Presence of any $\mathrm{MRI}$-erosion (erosion score $\geq 1$ ) and RA-specific erosion characteristics as identified previously (grade $\geq 2$ erosions, erosions in MTP5, erosions in MTP1 if aged $<40$ ) were studied with clinically apparent inflammatory arthritis development as outcome (median follow-up 17 months). Analyses were corrected for age, CRP, ACPA and MRI-detected inflammation

Results: Erosions were present in $20.6 \%$ of patients. Presence of erosions was not associated with arthritis development (HR multivariable analysis $0.85(95 \% \mathrm{Cl}$ $0.52-1.40)$ ). Also the different erosion characteristics were not predictive in CSApatients (grade $\geq 2 \mathrm{HR} 1.29$ (95\% Cl 0.40-4.14), erosions in MTP5 HR 0.89 (95\% $\mathrm{Cl} 0.38-2.09)$ and MTP1 if aged <40 HR 0.98 (95\% Cl 0.23-4.21)). MRl-erosions were more prevalent in ACPA-positive than in ACPA-negative patients $(32.3 \%$ versus $18.8 \%, p=0.02$ ). However, no association with arthritis development was observed in both subgroups.

Conclusion: MRI-detected erosions in hands and feet of patients with CSA were not predictive for arthritis development. These data warn against overinterpretation of MRI-detected erosions in CSA.

Disclosure of Interests: Fenne Wouters: None declared, Xanthe Matthijssen: None declared, Debbie Boeters: None declared, Robin ten Brinck: None declared, Annette van der Helm - van Mil Grant/research support from: The research lead ing to these results has received funding from the European Research Council (ERC) under the European Union's Horizon 2020 research and innovation programme (Starting grant, agreement No 714312) and from the Dutch Arthritis Foundation.

The funding source had no role in the design and conduct of the study., Ellis Niemantsverdriet: None declared

DOI: 10.1136/annrheumdis-2019-eular.2991

\section{LB0001 \\ EFFICACY AND SAFETY OF FILGOTINIB FOR PATIENTS WITH RHEUMATOID ARTHRITIS WITH INADEQUATE RESPONSE TO METHOTREXATE: FINCH1 PRIMARY OUTCOME RESULTS}

Bernard Combe $^{1}$, Alan Kivitz ${ }^{2}$, Yoshiya Tanaka ${ }^{3}$, Désirée van der Heijde ${ }^{4}$, Franziska Matzkies ${ }^{5}$, Beatrix Bartok ${ }^{5}$, Lei Ye ${ }^{5}$, Ying Guo ${ }^{5}$, Chantal Tasset ${ }^{6}$, John Sundy ${ }^{5}$, Neelufar Mozaffarian ${ }^{5}$, Robert B.M. Landewé ${ }^{7}$, Sang-Cheol Bae ${ }^{8}$, Edward C. Keystone ${ }^{9,10}$, Peter Nash ${ }^{11}$. ${ }^{1} \mathrm{CHU}$ Montpellier, Montpellier University, Montpellier, France; ${ }^{2}$ Altoona Center for Clinical Research, Duncansville, United States of America; ${ }^{3}$ University of Occupational and Environmental Health Japan, Kitakyushu, Japan; ${ }^{4}$ Leiden University Medical Center, Leiden, Netherlands; ${ }^{5}$ Gilead Sciences, Inc., Foster City, United States of America; ${ }^{6}$ Galapagos NV, Mechelen, Belgium; ; Amsterdam University Medical Center, Amsterdam, Netherlands; ${ }^{8}$ Hanyang University Hospital for Rheumatic Diseases, Seoul, Korea, Rep. of (South Korea); ${ }^{9}$ Mount Sinai Hospital, Toronto, Canada; ${ }^{10}$ University of Toronto, Toronto, Canada; ${ }^{11}$ University of Queensland, St. Lucia, Australia

Background: Filgotinib (FIL) is an orally administered, potent and selective inhibitor of Janus kinase 1 (JAK1) that has shown good efficacy and was well tolerated for treatment of rheumatoid arthritis (RA).

Objectives: To evaluate efficacy and safety of FIL treatment in patients with RA who have had an inadequate response to methotrexate (MTX). 